\title{
Dynamic maps: a visual-analytic methodology for exploring spatio-temporal disease patterns Denise A Castronovo ${ }^{1}$, Kenneth KH Chui ${ }^{2}$ and Elena N Naumova*2
}

\author{
Address: ${ }^{1}$ Mapping Sustainability, LLC, Jupiter, FL, USA and ${ }^{2}$ Department of Public Health and Community Medicine, Tufts University Medical \\ School, Boston, MA, USA \\ Email: Denise A Castronovo - denise@mappingsustainability.com; Kenneth KH Chui - kenneth.chui@tufts.edu; \\ Elena N Naumova* - elena.naumova@tufts.edu \\ * Corresponding author
}

Published: 30 December 2009

Environmental Health 2009, 8:61 doi:10.1 186/1476-069X-8-61

This article is available from: http://www.ehjournal.net/content/8/I/6I

(C) 2009 Castronovo et al; licensee BioMed Central Ltd.

This is an Open Access article distributed under the terms of the Creative Commons Attribution License (http://creativecommons.org/licenses/by/2.0), which permits unrestricted use, distribution, and reproduction in any medium, provided the original work is properly cited.
Received: 27 July 2009

Accepted: 30 December 2009

\begin{abstract}
Background: Epidemiologic studies are often confounded by the human and environmental interactions that are complex and dynamic spatio-temporal processes. Hence, it is difficult to discover nuances in the data and generate pertinent hypotheses. Dynamic mapping, a method to simultaneously visualize temporal and spatial information, was introduced to elucidate such complexities. A conceptual framework for dynamic mapping regarding principles and implementation methods was proposed.
\end{abstract}

Methods: The spatio-temporal dynamics of Salmonella infections for 2002 in the U.S. elderly were depicted via dynamic mapping. Hospitalization records were obtained from the Centers of Medicare and Medicaid Services. To visualize the spatial relationship, hospitalization rates were computed and superimposed onto maps of environmental exposure factors including livestock densities and ambient temperatures. To visualize the temporal relationship, the resultant maps were composed into a movie.

Results: The dynamic maps revealed that the Salmonella infections peaked at specific spatiotemporal loci: more clusters were observed in the summer months and higher density of such clusters in the South. The peaks were reached when the average temperatures were greater than $83.4^{\circ} \mathrm{F}\left(28.6^{\circ} \mathrm{C}\right)$. Although the relationship of salmonellosis rates and occurrence of temperature anomalies was non-uniform, a strong synchronization was found between high broiler chicken sales and dense clusters of cases in the summer.

Conclusions: Dynamic mapping is a practical visual-analytic technique for public health practitioners and has an outstanding potential in providing insights into spatio-temporal processes such as revealing outbreak origins, percolation and travelling waves of the diseases, peak timing of seasonal outbreaks, and persistence of disease clusters.

\section{Background}

The interactions between human health and physical environments are complex, for they involve many inter- acting variables that are geographical, chronological, and demographical. A good understanding of these interactions can lead to better strategized preventions and poli- 
cies. However, such understanding requires the ability to recognize, track, analyze and represent dynamic spatiotemporal processes $[1,2]$.

Infectious disease surveillance can benefit from the study of dynamic spatio-temporal processes through acquiring more information on three aspects: seasonality of the diseases, synchronization between diseases and exposures, and geographic distribution of diseases and exposures. Many infectious diseases exhibit distinct seasonal trends reflecting a temporal process that alternates between periods of low endemic levels with periods of outbreak. For instance, infection rates due to Salmonella spp. or Campylobacter jejuni peak in the summer while those due to Giardia and Cryptosporidium rise in autumn [3]. The temporal synchronizations of water and food-borne disease outbreaks and prevailing weather conditions suggest a strong influence asserted by the environment [4]. Spatially, the seasonal patterns of the diseases and their associations with environmental influences also vary across locations [5]. Understanding of dynamic spatio-temporal processes can organize help to organize the above variables into a systematic framework which would consequently spark new hypotheses. However, these analyses would require an innovative integration of geographic information and time-series data.

When applied to geographic information, visualization refers to the procedure of creating cartographic representations [6], or maps, of spatial data. A dynamic map is a series of "temporally-ordered snapshots of maps that each depicts a period in time" [7]. This tool introduces new insights to researchers into the spatio-temporal processes that would not be possible to demonstrate using static maps or statistical analysis $[8,9]$. When applied to infectious disease surveillance, dynamic maps show spatiotemporal information of a disease outbreak such as origins, percolation and travelling waves of disease incidence, peak timing of seasonal outbreaks, and persistence of disease clusters. Dynamic maps can also help researchers visually explore relationships through synchronization and overlay of outcome data with exposure variables [6].

The main objective of the study is to introduce dynamic mapping as a method for performing exploratory data analysis of disease dynamics and for generating testable hypotheses. We provide a series of dynamic map examples that overlay Salmonella-related hospitalization data for the U.S. elderly (aged 65 and over) onto maps of environmental exposure factors such as livestock, ambient temperature and ambient temperature deviance from the 30 -year norm. The secondary objective is to introduce principles for building effective dynamic maps for disease outcome data, which are discussed herein.

\section{Methods}

The methodological description consists of four parts: (i) abstraction and aggregation of the outcome data, (ii) abstraction, aggregation, and mapping of the exposure data, (iii) static mapping of the outcome data, and (iv) composition of the dynamic map. Details of these four steps are as follows.

\section{Health outcome data--abstraction and aggregation}

Hospitalization records for Salmonella infections were abstracted from the Centers for Medicare and Medicaid Services (CMS) for all Medicare recipients aged 65 or above in the contiguous U.S. for 2002 (Alaska, Hawaii, Virgin Islands and Puerto Rico were excluded from the analysis). In the U.S., more than $95 \%$ of the population aged 65 or above are covered by Medicare; hence the CMS records are nationally representative. Each hospitalization record contains information on the date of admission, ZIP code and state of residence, age and up to 10 diagnostic codes based on the International Classification of Diseases, Ninth Revision, Clinical Modification (ICD-9-CM). Records containing any diagnosis starting with "003", indicating "other Salmonella infections", were included in this analysis. Conditions starting with "002", indicating "typhoid and paratyphoid fevers" caused by Salmonella typhi infection, were excluded due to their low frequencies.

Temporally, all records were aggregated to a monthly level; spatially, to a county level. Monthly hospitalization rates were computed using county population aged 65 or above as the denominator. Population counts were obtained from the Census 2000, Summary File 1. To minimize spurious high rates caused by extremely low denominators, a spatial re-aggregation scheme [see Figure 1] was applied to incorporate counties with a low number of elderly into the adjacent counties until the total number of elderly exceeded 1,000, a parameter that was selected based upon the population of elderly per county and the frequency of diseases. The re-aggregation collapsed the number of counties within the continental U.S. from 3,109 to 2,794. On the maps, the boundaries between two aggregated counties were removed to form one larger area. For each aggregated county, the elderly population and monthly Salmonella hospitalization counts were summed together. Monthly hospitalization rates per 10,000 elderly, $\mathrm{R}_{i j}$, were calculated for each aggregated county i $(i=1: 2,794)$ and month $j(j=1: 12)$ as follows:

$\mathrm{R}_{\mathrm{ij}}=\left[(\text { Monthly Salmonella count })_{\mathrm{ij}} /(\text { Total elderly population })_{\mathrm{i}}\right]^{*} 10,000$

\section{Exposure data--abstraction, aggregation and mapping}

Ambient temperature data were obtained from the PRISM Group at Oregon State University for average maximum 


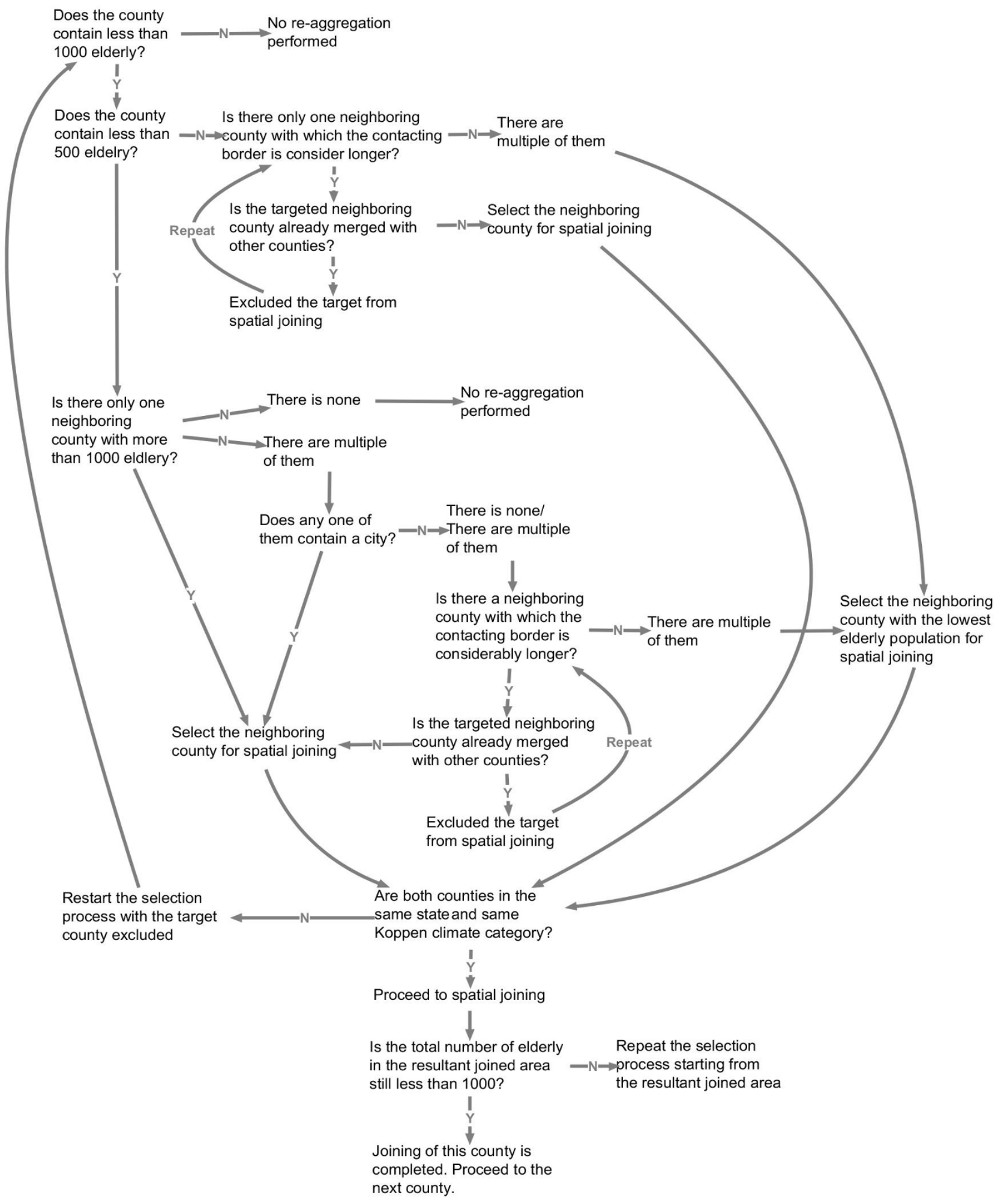

Figure I

The re-aggregation scheme. The chart explains the chain of decisions made when aggregating counties with low elderly population in this study. Arrows with "Y" indicates "Yes"; "N" indicates "No". 
monthly temperature, average maximum annual temperature, and average maximum temperature anomaly for 2002. Data were downloaded in ESRI ArcInfo Ascii Grid format with a spatial resolution of 4 kilometer grid cells and imported into ESRI's ArcMap software. The aggregated county polygons were overlaid onto each temperature grid and the mean temperature or temperature anomaly was calculated for each county. Choropleth maps for the average monthly and annual maximum temperature were created by considering the full range of county-level temperature values with the classification emphasizing temperatures above freezing shown in shades of yellow to dark orange. The monthly temperature anomaly maps show the deviation in temperature on a monthly basis from the 30-year norm for each month. The choropleth maps for the temperature anomaly emphasize temperature deviations greater than $+3^{\circ} \mathrm{C}$ in pink and less than $-2^{\circ} \mathrm{C}$ in purple.

Exposure data on livestock were obtained from the 2002 quinquennial Census of Agriculture gathered by the U.S. Department of Agriculture's National Agricultural Statistics Service. County-level data on the number of broiler and other meat-type chickens sold from livestock farms to food distributors were downloaded and mapped by matching the county FIPS codes with county-level polygons. A choropleth map of the number of broiler chickens sold in 2002 was created by first performing a logarithmic transformation on the data to alleviate the problematic skewness, followed by assigning the data into five categories using a natural breaks classification.

\section{Mapping of the health outcome}

Twelve monthly static maps for 2002 were created. To facilitate visual comparison between months, the bin sizes adopted in the legend were unified across the 12 maps. Countywide Salmonella hospitalization data for the elderly were extremely skewed due to a disproportionately high amount of low rates and a small amount of high rate outliers. Seasonal changes further exaggerated the problem since there was a two to three-fold increase in salmonellosis during the summer time. To alleviate the problems caused by the skewness, a logarithmic transformation was applied. The logarithmic transformation helps to optimally assign categories into high and low brackets (Figure 2). The unified bin sizes were derived by assigning a natural break classification scheme to the month with the highest amount of hospitalization cases. The resulting classification scheme was then applied to the other maps.

Hospitalization rates were represented on the map with graduated dot symbols. The graduated colour and symbol size of the dots allow for a quick visual comparison between high and low rates; larger and darker dots indicate higher rates and smaller and lighter dots indicate lower rates. The sizes of the graduated dot symbols are proportional to the outcome rates and hence are preferred over polygons with graduating shades, which are harder to interpret due to the wide range of county sizes. The size of the largest graduated dot symbol was determined using the average county size so that overlapping of dots and county boundaries were minimized.

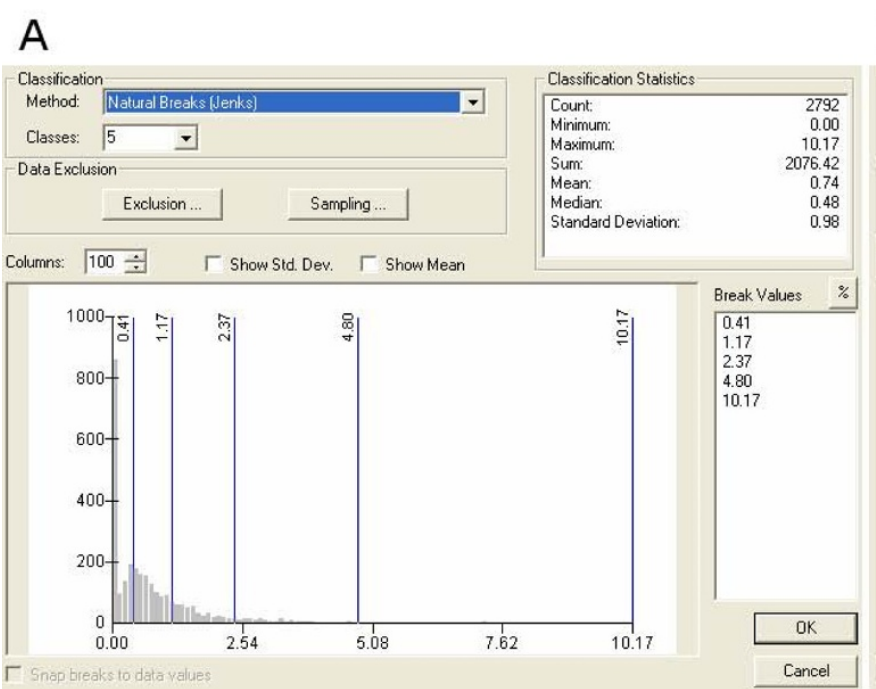

\section{B}

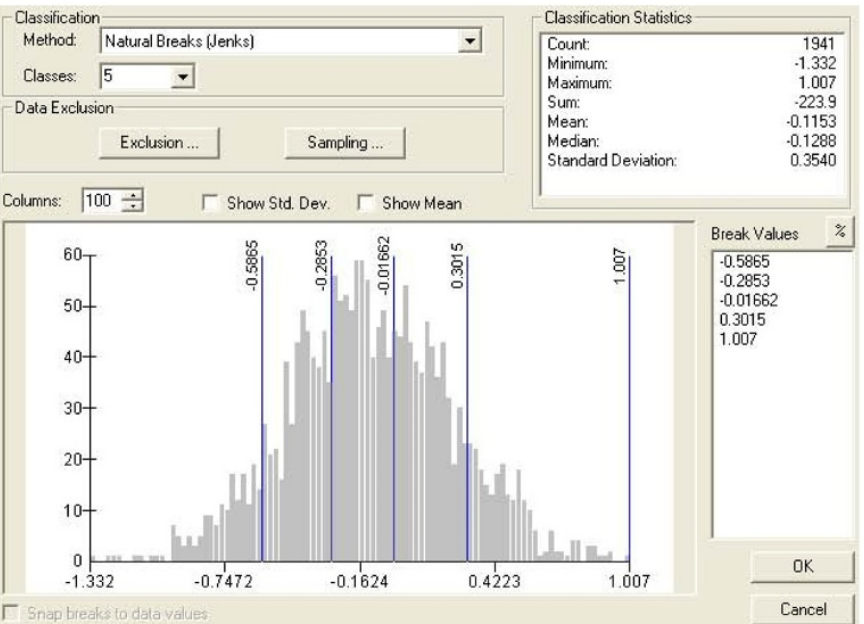

\section{Figure 2}

Natural breaks classification using logarithmic transformation. A. Natural breaks classification on untransformed Salmonella hospitalization rate data, B. Natural breaks classification on log transformed Salmonella hospitalization rate data. 


\section{Dynamic Mapping}

The monthly maps of county-level Salmonella-related hospitalizations were superimposed onto maps of environmental exposures including: average monthly maximum temperature, the number of broiler chickens sold in 2002, and monthly temperature deviation from the 30-year norm. Maps were exported from ArcMap and imported into Adobe Flash software as an individual key frame. The frames were sequentially incorporated into a movie.

The speed for playing the map movie was optimized so that that the viewers can have enough time to identify clusters and commit the frame to short memory, allowing them to distinguish changes when the next frame appears. Herein, the dynamic maps are shown at a frame rate of one frame per second and the Salmonella infection data span two key frames. An interactive interface consisting of control buttons for stop, play, move forward or back one frame, and replay are added to help the viewers investigate the dynamic map at their own pace.

To indicate the calendar time, a label with the year and month was incorporated into the maps. For maps that span more than one year, a sliding calendar bar was added to help the viewer track time duration. The sliding calendar bar could also be interactive so that the viewer could easily navigate to a specific point in time.

Narrations were incorporated into the movies to help the viewers appreciate the dynamics of Salmonella hospitalizations with respect to the exposures.

\section{Results}

In 2002, there were a total of 1,686 hospitalizations associated with Salmonella infections. The annual rate was 4.85 hospitalizations per 100,000 elderly (aged 65 or above). The infections were more common during the summer and early fall season: $64 \%$ of the cases occurred between May and October. For illustrative purposes, Dynamic Map 1 [see Additional file 1] shows the monthly rate of Salmonella-related hospitalizations for the U.S. elderly superimposed on a static map of average annual temperature for 2002. The map shows an association between the average-annual temperature gradient, latitude, and longitude. In the Great Plains where elevation is relatively flat there was an overall north to south trend of low to high temperatures. Topography such as higher elevation is also associated with average temperature, as suggested by the Appalachian and Rocky Mountain regions that have lower temperatures. The Northeastern coastal areas experienced warmer temperatures than farther inland.

Salmonella-related hospitalizations were most prevalent during the months of July and August, which is consistent with previous studies demonstrating that hot and humid weather is favorable for Salmonella survival and replication [3]. The summer is also associated with outdoor cooking where food handling precautions are often inadequate, thereby increasing the risk for individuals to acquire foodborne infections like Salmonella [10]. In July, the majority of the hospitalizations occurred in the South, particularly in Mississippi where an apparent cluster existed. One might expect higher counts of salmonellosis in urban areas, but the map demonstrates clusters of low rates in the New York metropolitan area.

Dynamic Map 2 [see Additional file 2] shows the monthly rate of Salmonella-related hospitalizations for the U.S. elderly in 2002 superimposed on a dynamic map of average monthly maximum temperature. The map indicates that latitude and longitude are not the best predictors of temperature change due to topography, proximity to the coast, the jet stream and dominant wind patterns. In July, average monthly maximum temperatures less than $90^{\circ} \mathrm{F}$ $\left(32.2^{\circ} \mathrm{C}\right)$ corresponded with higher elevations and predominant wind patterns from the jet stream. Salmonella infections peaked during July and August, corresponding with average monthly temperatures greater than $83.4^{\circ} \mathrm{F}$ $\left(28.6^{\circ} \mathrm{C}\right)$.

By overlaying Salmonella-related hospitalizations with data of temperature anomaly, as demonstrated in Dynamic Map 3 [see Additional file 3], viewers can determine whether the rates increased when temperatures were significantly higher than the 30-year norm: an indication of a heat wave induced outbreak. Areas on the map shown in orange to pink were warmer than normal and purple areas were colder than normal. In July the cluster of high rates in Mississippi corresponded with temperatures 0 to $3.7^{\circ} \mathrm{F}\left(0\right.$ to $\left.-2.1^{\circ} \mathrm{C}\right)$ colder than normal. In August, the cluster of salmonellosis in the New York metropolitan area corresponded with temperatures higher than normal of 3.8 to $5.4^{\circ} \mathrm{F}\left(2.1\right.$ to $\left.3.0^{\circ} \mathrm{C}\right)$.

Dynamic Map 4 [see Additional file 4] shows the monthly rate of Salmonella-related hospitalizations for the U.S. elderly in 2002 superimposed on a map showing the number of broiler chickens sold in 2002 from livestock farms to food distributors. The number of chickens sold per county may be associated with Salmonella hospitalizations in the elderly through direct or indirect exposures. The elderly could be directly exposed to Salmonella if they work in the chicken industry, or they could be indirectly exposed if the livestock fecal matter contaminates drinking water sources. The dynamic map helps to look for associations between exposure and Salmonella incidence. Dynamic Map 4 illustrates that Salmonella-related hospitalizations corresponded with high numbers of broiler chickens sold in July in Mississippi and Maryland, in August in North Carolina, and in October in Oklahoma. 


\section{Discussion \\ The features of dynamic maps}

Dynamic maps provide additional insight into temporal disease dynamics that is not obtainable from static maps. In an exploratory context, static maps can be used to identify and compare patterns in a spatial context only. Static maps allow us to determine what patterns exist, where they exist, how they exist, and identify how they compare to other spatial patterns. Dynamic mapping deepens our understanding of the data by adding the temporal domain $[6,11]$, through which researchers can further investigate when and where diseases emerge, when and to where they spread, for how long or where they persist, and identify differences in their spatio-temporal patterns. Percolation of Salmonella infections was demonstrated in the dynamic maps where Salmonella-related hospitalizations showed up in northern Mississippi in May and June and spread to southern Mississippi in July. A persistent cluster of Salmonella-related hospitalizations occurred in the New York metropolitan area from May through October with an apparent peak in August.

Dynamic maps allow us to investigate processes, such as disease dynamics, rather than simply recognize static patterns: it is useful for modeling disease transmission, place of exposure and probabilities of hospitalization. By overlaying disease outcome with exposure variables, multiple data sets can be integrated to identify trends, degree of synchronization [6] between exposures and outcomes, or even synchronization between two outcomes such as mortality and co-morbidity. Furthermore, dynamic mapping allows for the visualization of disease incidences and seasonal patterns at conventional temporal scales such as daily, weekly or monthly, which would be difficult to convey through traditional static maps. Dynamic Map 2 [Additional file 2] illustrates the seasonal peaks in temperature during the summer months with the hottest temperatures occurring in the South and moving northward. In concurrence with the temperature upsurge, Salmonella hospitalizations spread northward from July to August, demonstrating a travelling wave with a potentially strong influence from environmental factors such as temperature and humidity.

Studies have shown that peaks in ambient temperature correspond with peaks in Salmonella incidence with a lag of one to five weeks $[4,5]$. By superimposing monthly Salmonella hospitalizations onto maps of ambient temperatures and livestock abundances, dynamic maps can be used to generate hypotheses about routes of exposure, spatial patterns of transmission, and diseases clustering. When viewing the dynamic maps of monthly Salmonella infections superimposed onto monthly temperatures the rationale for exploration of a lagged relationship between exposure and outcome emerges. If a lag relationship does exist, dynamic maps offer channel to observe the synchronization between the outcome and exposure.

Dynamic maps allow viewers to track multiple locations of disease occurrence simultaneously. The U.S. Centers for Disease Control and Prevention (CDC) reported minor outbreaks of Salmonella during 2002. From January through April of 2002, there were 47 cases of multi-drug resistant Salmonella Newport in five states: New York, Michigan, Pennsylvania, Ohio and Connecticut, causing 17 hospitalizations [12]. From March through May of 2002, tainted cantaloupe from Mexico caused 58 cases of Salmonella Serotype Poona infections in 10 states: California, Washington, Oregon, Colorado, Nevada, Missouri, Texas, Arkansas, Minnesota, and Vermont. Eleven cases were in individuals aged $>60$ years and there were 10 hospitalizations reported [13]. In June 2002, an outbreak of Salmonella Serotype Javiana infected people who attended an athletic competition in Orlando, Florida [14]. There were 141 ill people dispersed across 32 states with 3 known hospitalizations. These outbreak examples illustrate that one strain of Salmonella may result in a multistate outbreak that affects nonadjacent states. Also, the outbreak may originate in one location but be reported in multiple states depending upon where the infected patient lives. Dynamic maps provide users with a visualanalytic capability that allows more careful consideration of the nuances within the data.

It is important to recognize that the major strength of the proposed dynamic mapping methodology is its visualanalytic capabilities to assist as an exploratory tool to catalyze the scientific process by propagating potential research questions and future directions. While the methodology is not yet ready to test hypotheses, this capability would be the next step for methodological development.

\section{Designing dynamic maps}

Determining class breaks for dynamic maps poses a significant challenge because a single scheme must work for multiple maps and the placement of a class break can either emphasize small fluctuations in the data or mask large changes [15]. Monmonier [7] uses a bin scoring method to assign category breaks to dynamic spatio-temporal choropleth maps in order to avoid creating a visually noisy and constantly changing display that could confound the visualization of major trends in the data. Harrower [15] puts forth a methodology that avoids classification altogether by placing the highest and lowest values at each end of a continuous colour ramp and placing the remaining data proportionally along that continuum for each map frame. His study shows that this unclassed choropleth dynamic mapping method can improve our ability to see gradual changes in the mapped data which more realistically reflect the underlying process. After the 
class break schema is determined, dynamic maps are built by producing individual map frames one-by-one for each point in time [16] using GIS and animation software [17].

When we developed our principles for effective dynamic mapping, we heavily borrowed from Harrower's [18] principles including: (1) create a simple base map with only a few data classes or features so that it is quickly readable, (2) provide map controls to stop and playback the movie frame-by-frame, (3) direct the viewer's attention with voice over or dynamic map symbols, (4) generalize the map data to highlight important trends, (5) use categorical data legends such as "low", "medium" and "high", and (6) provide a short introduction to the interface before showing the data to break up the learning curve.

\section{Seven principles for effective dynamic mapping}

Many factors should be considered when composing effective dynamic maps for disease incidence data. The following seven principles were suggested for consideration.

\section{Principle I}

Choose the optimal aggregation scheme. One important consideration for dynamic mapping of seasonal disease outcome data is to select a temporal scale that is small enough to pick up the seasonal signal while large enough to be easily compiled. Choosing a temporal scale that is too small could cause too many sporadic changes in the dynamic maps that would prevent the map reader from seeing the overall seasonal trend, especially since the viewers' visual memory from one frame to the next is extremely limited. In the examples, countywide Salmonella-related hospitalization counts for the elderly were so low that the data had to be aggregated to a monthly level to show seasonal patterns and reduce noise.

The dynamic mapping methodology is best suited for temporal data with a high frequency such as daily, weekly, or monthly occurrences. With each level of data aggregation there is a trade-off between what is gained in showing overall trends and what is lost in terms of visualizing the dynamic properties of the disease (e.g. outbreak origins, persistence, percolation, travelling waves, and peak timing). Recognizing this trade-off is especially important when studying the spatio-temporal dynamics of highly communicable diseases or diseases with very short incubation periods, such as influenza. Researchers should optimize the spatio-temporal scale according to their expertise.

\section{Principle 2}

Choose the correct colour scheme and legends. For the temporal information to be efficiently transferred, cartographers should minimize the viewers' effort on locating and comparing the key information on the dynamic maps. For instance, adding a dark background colour behind the map helps the user focus on the data represented by the brighter colours. The classification schemes used in the legend should also be unified so that a certain hue, saturation, brightness, transparency, and size represent the same information across the frames in a set of dynamic maps. When overlaying health outcome data onto exposure data, the colour scheme for outcome should be distinguishable from that of the exposure data. Complimentary colours (colours that are opposite to each other on a colour wheel) work well with the exception of reds and greens, which are not distinguishable among viewers with colour-blindness. Colour gradations need to be different enough so that each one is distinguishable at a quick glance. By changing the colour saturation and value, colours are easily made lighter or darker within the same hue.

\section{Principle 3}

Choose the correct frame speed. In this study the frame speed was optimized to 1 frame/second. However, the speed can be adjusted depending on the size of the map and complexity of the data. When viewing the maps, it is best to view them for at least three times. The first viewing focuses on understanding the exposure variable in the background. The second viewing entails understanding the outcome variable and its spatial patterns. In the third round the viewers can look for relationships between exposure and outcome, clusters of health outcomes, and percolation.

\section{Principle 4}

Think like the viewers. The usefulness of dynamic maps for environmental and public health research depends heavily on the cartographer's ability to make the maps simple, informative and attractive [18]. Morrison [19] and Harrower [18] discussed four challenges associated with watching and learning from dynamic maps: 1) disappearance--the maps change dramatically from moment to moment causing the map readers to miss information, 2) attention--map readers are confused by the map interface or do not know where to look on the map, 3) complexity-many maps have too much information which confounds their message, and 4) confidence--viewers are less confident in the knowledge they acquire from dynamic maps due to inexperience with them. Our Salmonella dynamic map examples used several principles identified by Harrower [18] to help overcome these challenges. To overcome issues of disappearance, the maps used a simple base map, colour-blindness friendly colour schemes, and map playback tools that allow the user to watch the animation many times, stop it and continue frame-by-frame. To reduce the complexity of the maps, the monthly data were filtered to include only a few months prior to and 
after the seasonal peak so that periods with low endemic levels of Salmonella infections were not included.

\section{Principle 5}

Incorporate control interface. Interaction with dynamic maps, such as building in a set of buttons that control the flow of the maps, allows users to pace their learning.

\section{Principle 6}

Understand the limitations of dynamic maps. Dynamic maps represent a delicate balance between being useful versus incomprehensible from cognitive and perceptual information overload $[20,21]$. Since the frames of animated maps change frequently, animated maps are useful for gaining an overall perspective of spatio-temporal processes rather than emphasizing specific rates for specific locations and time periods $[7,8,18]$. According to Harrower [21], 'the problem is not that map viewers are incapable of seeing the changes occurring on the animated map, it is that they have well-documented trouble remembering what they saw and integrating it into their knowledge schemata.' One of the key research challenges for space-time visualization is to understand the types of problems that are best suited for animated maps $[21,22]$.

\section{Principle 7}

Be open to improvement. Dynamic maps require careful interpretation, especially when they are applied to outcome data with low counts. Visual clusters of hospitalization rates need to be further explored into the potential number of cases because sometimes high rates are created from single hospitalizations in areas with a small elderly population. The CDC defines an outbreak of foodborne illness as a cluster of two or more infections caused by the same pathogen and linked to the same food [23]. In this study, we attempted to alleviate this problem by introducing the re-aggregation scheme. The versatile functions in GIS software should be thoroughly explored and implemented in order to compensate inherent shortcomings in the data that can potentially mislead the viewers.

\section{Conclusion}

Dynamic mapping provides a practical visualization tool to examine seasonality in infectious disease and its relationship to environmental factors. This paper applied the approach to study Salmonella-related hospitalizations nationwide in the U.S. elderly population for the 2002 season and demonstrated a framework that can stimulate hypothesis generation for better understanding disease seasonality. As an extension of our earlier work [24], fundamental principles for making effective dynamic maps were proposed. These rules serve as an intuitive guideline for properly constructing a graphical display that contains a well-understood statistical context or logical path, helping to explain data or concepts by taking advantage of vis- ual perceptions, and objectively displaying the results and concepts in a manner that highlights the unexpected and motivates questions.

Understanding the nature of environmental exposures, the temporal features of disease manifestation, and the linkages between health outcomes and the environment can be substantially enhanced by modern visual-analytical tools. Research in the fields of environmental epidemiology would benefit from the proposed approach of dynamic mapping where data mining and exploration, hypothesis building and testing are required.

\section{List of Abbreviations}

CDC: Centers for Disease Control and Prevention; CMS: Centers for Medicare and Medicaid Services; FIPS code: Federal Information Processing Standard code; GIS: Geographic Information System; ICD-9-CM: International Classification of Diseases, Ninth Revision, Clinical Modification; PRISM: Preparation for Instruction of Science \& Math; U.S.: The United States; ZIP code: Zoning Improvement Plan code

\section{Competing interests}

The authors declare that they have no competing interests.

\section{Authors' contributions}

DAC created the dynamic maps and drafted the manuscript. KKHC drafted the manuscript and created Figures. ENN conceived the study and participated in its design and coordination. All authors read and approved the final manuscript.

\section{Additional material}

\section{Additional file 1}

Dynamic Map 1. Monthly rate of Salmonella hospitalizations for the U.S. elderly ages 65 and over superimposed on the average temperature for the year 2002.

Click here for file

[http://www.biomedcentral.com/content/supplementary/1476069X-8-61-S1.SWF]

\section{Additional file 2}

Dynamic Map 2. Monthly rate of Salmonella hospitalizations for the U.S. elderly ages 65 and over superimposed on the average monthly maximum temperature.

Click here for file

[http://www.biomedcentral.com/content/supplementary/1476069X-8-61-S2.SWF] 


\section{Additional file 3}

Dynamic Map 3. Monthly rate of Salmonella hospitalizations for the U.S. elderly ages 65 and over superimposed on the temperature deviation from the 30-year norm.

Click here for file

[http://www.biomedcentral.com/content/supplementary/1476069X-8-61-S3.SWF]

\section{Additional file 4}

Dynamic Map 4. Monthly rate of Salmonella hospitalizations for the U.S. elderly ages 65 and over superimposed on number of broiler chickens sold in 2002.

Click here for file

[http://www.biomedcentral.com/content/supplementary/1476069X-8-61-S4.SWF]

\section{Acknowledgements}

We thank the National Institute of Environmental Health Sciences who provided funding for this study as part of the "Gastroenteritis and Extreme Weather Events in Elderly" Project (GEWEL, OIESOI3I7I) and also the National Institute of Allergy and Infectious Disease grant entitled, "Generation and Decay of Memory T Cells in Young, Old and Immunocompromised Populations" (NOI HHSN266200500032).

\section{References}

I. Harrower M, Fabrikant SI: The role of map animation for geographic visualization. In Geographic Visualization Edited by: Dodge M, McDerby M, Turner M. New Jersey: John Wiley \& Sons, Ltd; 2008:49-65.

2. Yattaw NJ: Conceptualizing Space and Time: A Classification of Geographic Movement. Cartography and Geographic Information Science 1999, 26:85-98.

3. Naumova EN, Jagai JS, Matyas B, DeMaria A, MacNeill IB, Griffiths JK: Seasonality in six enterically transmitted diseases and ambient temperature. Epidemiology and Infection 2007, 135:28I-292.

4. Hald T, Andersen JS: Trends and seasonal variations in the occurrence of Salmonella in pigs, pork and humans in Denmark, 1995-2000. Berliner Und Munchener Tierarztliche Wochenschrift 200I, I I 4:346-349.

5. Kovats RS, Edwards SJ, Hajat S, Armstrong BG, Ebi KL, Menne B, Grp $C$ : The effect of temperature on food poisoning: a timeseries analysis of salmonellosis in ten European countries. Epidemiology and Infection 2004, I 32:443-453.

6. Blok C, Kobben B, Cheng T, Kuterma A: Visualization of relationships between spatial patterns in time by cartographic animation. Cartography and Geographic Information Science 1999, 26:139-15I.

7. Monmonier M: Minimum-change categories for dynamic temporal choropleth maps. Journal of the Pennsylvania Academy of Science 1994, 68:42-47.

8. Dorling D, Openshaw S: Using computer animation to visualize space-time patterns. Environment and Planning B: Planning and Design 1992, 19:639-650.

9. Harrower M: Cartographic Animation. In International Encyclopedia of Human Geography Edited by: Kitchin R, Thrift N. forthcoming: Elsevier Press; 2007 in press.

10. Naumova EN: Mystery of seasonality: getting the rhythm of nature. J Public Health Policy 2006, 27:2-12.

11. MacEachren AM: How Maps Work: Representation, Visualization, and Design Ist edition. New York: The Guilford Press; 1995.

12. CDC: Outbreak of multidrug-resistant Salmonella newportUnited States, January-April 2002. MMWR Morb Mortal Wkly Rep 2002, 51:545-548.

13. CDC: Multistate outbreaks of Salmonella serotype Poona infections associated with eating cantaloupe from Mexico-United States and Canada, 2000-2002. MMWR Morb Mortal Wkly Rep 2002, 5 I: 1044-1047.
14. CDC: Outbreak of Salmonella serotype Javiana infections-Orlando, Florida, June 2002. MMWR Morb Mortal Wkly Rep 2002, $51: 683-684$.

15. Harrower M: Unclassed Animated Choropleth Maps. Cartographic Journal, The 2007, 44:313-320.

16. Ormeling F: Teaching Animated Cartography. In Proceedings of the Seminar on Teaching Animated Cartography; Escuela Universitaria de Ingeniera Tecnica Topografica, Madrid, Spain Edited by: Ormeling F, Köbben B, Gomez RP. ICA, Enschede; 1995:21-29.

17. Harrower M: A look into the history and future of animated maps. Cartographica 2004, 39:33-42.

18. Harrower M: Tips for designing effective animated maps. Cartographic Perspectives 2003, 44:63-65.

19. Morrison J: Does animation facilitate learning? An evaluation of the congruence and equivalence hypothesis. Stanford University, Department of Psychology; 2000.

20. Harrower M: The cognitive limits of animated maps. Cartographica 2007, 42:269-277.

21. MacEachren AM: Research challenges in geovisualization. Cartography and Geographic Information Science 200I, 28: I-II

22. Slocum TA, Blok C, Jiang B, Koussoulakou A, Montello DR, Fuhrmann $S$, Hedley NR: Cognitive and usability issues in geovisualization. Cartography and Geographic Information Science 200I, 28:6I-75.

23. CDC: OutbreakNet Team Overview. [http://www.cdc.gov/out breaknet/].

24. Naumova EN, O'Neil E: Statistical graphics: Philosophy to the rescue! Annual Joint Statistical Meetings of the American Statistical Association; August 5th-9th; Atlanta, Georgia 200I.

Publish with Bio Med Central and every scientist can read your work free of charge

"BioMed Central will be the most significant development for disseminating the results of biomedical research in our lifetime. "

Sir Paul Nurse, Cancer Research UK

Your research papers will be:

- available free of charge to the entire biomedical community

- peer reviewed and published immediately upon acceptance

- cited in PubMed and archived on PubMed Central

- yours - you keep the copyright
BioMedcentral 